\title{
A novel in vivo technique for endoscopic visualization of aortic valve motion and inner wall of large arteries for immediate postoperative direct observation
}

\author{
Takehiro Yamakoshi $^{*}$, Shinobu Tanaka ${ }^{2}$, Hiroshi Ohtake ${ }^{3}$, Go Watanabe ${ }^{3}$ and Ken-ichi Yamakoshi ${ }^{2,4}$ \\ ${ }^{1}$ Information and Systems Engineering, Graduate School of Engineering, Fukuoka Institute of Technology, 3-30-1 Wajiro-higashi, Higashi-ku, Fukuoka-shi, \\ Fukuoka 811-0295, Japan \\ ${ }^{2}$ Graduate School of Natural Science and Technology, Kanazawa University, Kakuma-machi, Kanazawa-shi, Ishikawa 920-1192, Japan \\ ${ }^{3}$ NewHeart Watanabe Institute, 3-19-11 Hamadayama, Suginami-ku, Tokyo 168-0065, Japan \\ ${ }^{4}$ Showa University School of Medicine, 1-5-8 Hatanodai, Shinagawa-ku, Tokyo 142-8555, Japan
}

\section{Background}

Coronary and pulmonary angioscopy capable of allowing observation of the lumen of small arteries, and intravascular ultrasound (IVUS) as well for obtaining images of the vasculature, are useful for clinical vascular diagnosis. A vascular endoscope incorporating a balloon to block off coronary blood flow has been reported to be particularly helpful in minimally invasive endovascular therapy [1]. The use of an endoscope to observe cardiac cavities is also of interest and several studies to realize this possibility toward clinical use have been tried using animal experiments [2,3]. An ex vivo study [4] was recently reported to assess clinical availability of an intravascular endoscopy improvement through narrow-band imaging [5] using extracted porcine vessels (aortic, carotid and femoral arteries) with simulated defects such as mechanical and thermal ablation. Although this ex vivo study presented endoscopic intra-arterial wall images taken just after being flushed with blood, it is difficult to acquire such images under an in vivo condition, and naturally to obtain an aortic valve motion as well. "Balloon aortoscopy [6]", on the other hand, is a unique technique to observe in vivo inner wall of aorta via transparent balloon wall. However, during the observation period, the aortic blood flow is blocked off by the inflated balloon. In view of these findings, it is desirable to acquire the direct in vivo observation of the motion of the aortic valve and the inner walls of large arteries.

\section{Objective}

The aim of the research described in the present paper is therefore to describe an initial development to realize such visualization technique without blocking off blood flow.

\section{Methodology}

Figure 1 is a schematic representation showing basic concept of intravascular endoscope. A jet is produced by a saline tank (capacity: 5 letters, max. pressure: $0.6 \mathrm{MPa}$ ) pressurized by a conventional air cylinder. At the outlet port of the tank, a high-speed solenoid valve (A2013, Precision Dynamics Co. Ltd., Valencia, USA) is connected so as to control timing and amount of saline injection. Approximately $1 \mathrm{ml}$ (less than $2 \mathrm{ml}$ ) of saline is discharged each cardiac cycle, synchronized to the diastolic phase using electrocardiogram (ECG), which can displace blood from the local area to be observed. Consecutive images
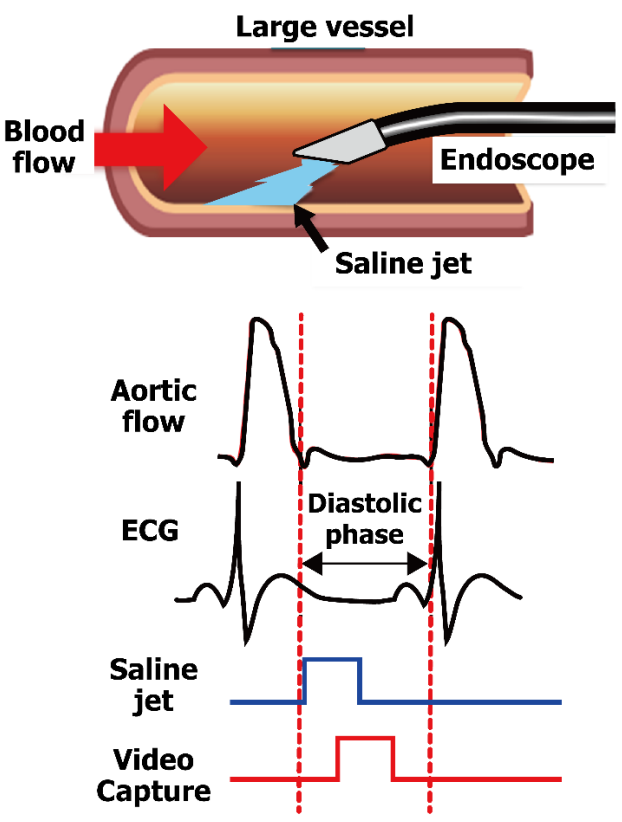

Figure 1. Schematic representation showing basic concept of endoscopic visualization (Modified by Figure 1 in the proceedings of the $33^{\text {th }}$ Ann Int Conf of the IEEE Eng Med Biol Soc)

of this area obtained instantaneously via an image-guide in each cardiac cycle are conflated to create a video picture. Based on this principle, Figure 2(a) shows a developed prototype, $6.2 \mathrm{~mm}$ diameter, vascular endoscope, having channels for light-/image-guide (1.65/1.45

${ }^{*}$ Correspondence to: Takehiro Yamakoshi, $\mathrm{PhD}$, Associate Professor, Information and Systems Engineering, Graduate School of Engineering, Fukuoka Institute of Technology, 3-30-1 Wajiro-higashi, Higashi-ku, Fukuoka-shi, Fukuoka 8110295, Japan, Tel: 810926065704; E-mail: yamakoshi@fit.ac.jp

Key words: endoscopic visualization, aortic valve motion, large arterial observation, vascular stenosis, grafting, vascular imaging

Received: July 23, 2018; Accepted: August 10, 2018; Published: August 13, 2018 
$\mathrm{mm}$ in diameter) optical fibers, forceps $(2.0 \mathrm{~mm}$ diameter; for the use of endovascular intervention, if needed) and the jet (1.72 $\mathrm{mm}$ diameter). A hemo-visor was fixed on the tip of the endoscope so as to reduce blood inflow around the area to be flushed with blood (Figure 2(b)).

Prior to clinical application, animal evaluation was carried out using 8 anesthetized pigs (30-40 kg body weight), with the endoscope cannulated into the aorta via a left iliac artery. The tip of the endoscope was firstly placed on the ascending aorta nearly close to the aortic valve and then withdrawn gradually until the tip was positioned on the abdominal aorta. In order to endoscopically observe postoperative conditions of indwelling of a stent and implantation of vascular prosthesis, a surgical operation was conducted to set a stent in the abdominal artery in some pigs and was also carried out to replace a synthetic vascular graft in the abdominal artery in the other pigs.

\section{Results}

It has been possible to capture clear images of the aortic valve cusp (Figure 3(A) and web-video (a)), the branching configuration in the aortic arch (Figure 3(B) and web-video (b)), the fixation of a stent (Figure 3(C) and web-video (c)) and the replacement of a synthetic vascular graft in the abdominal aorta (Figure 3(D) and web-video (d)) after the surgical procedure.

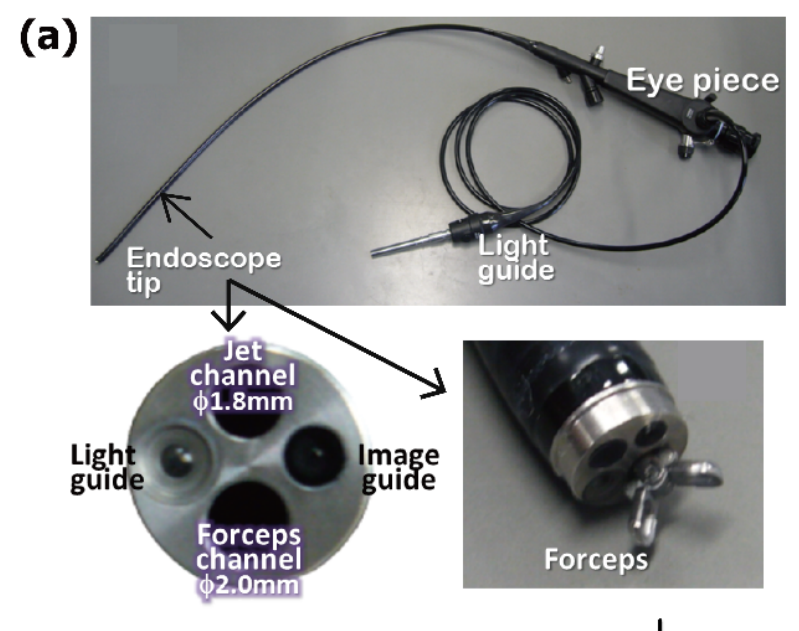

(b)

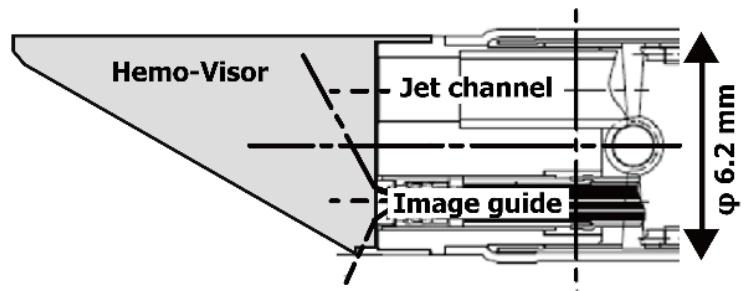

Figure 2. Photographs of the newly designed endoscope (a) and details of the tip of the endoscope (b). (Modified by Figure $2 \& 3$ in the proceedings of the $33^{\text {th }}$ Ann Int Conf of the IEEE Eng Med Biol Soc)
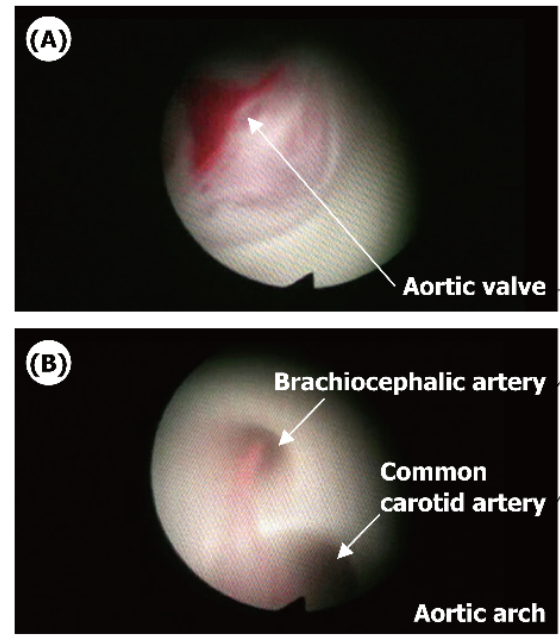

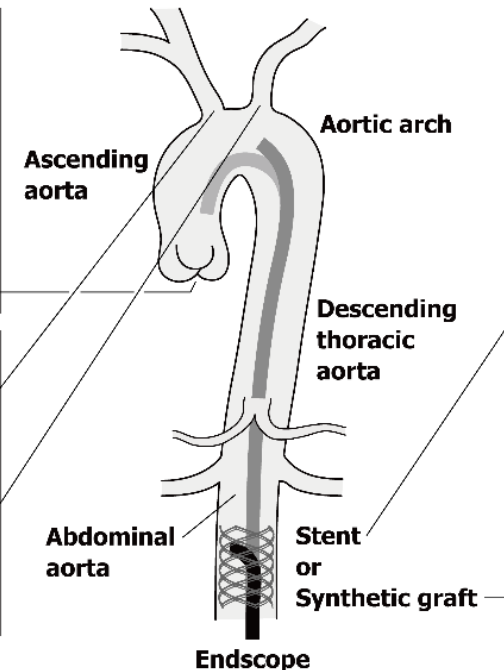

Figure 3. Images of aortic valve (A), branching configuration of the aortic arch (B), edge of a stent set in the abdominal artery (C) and suture site of a synthetic vascular graft replaced in the abdominal artery (D) 


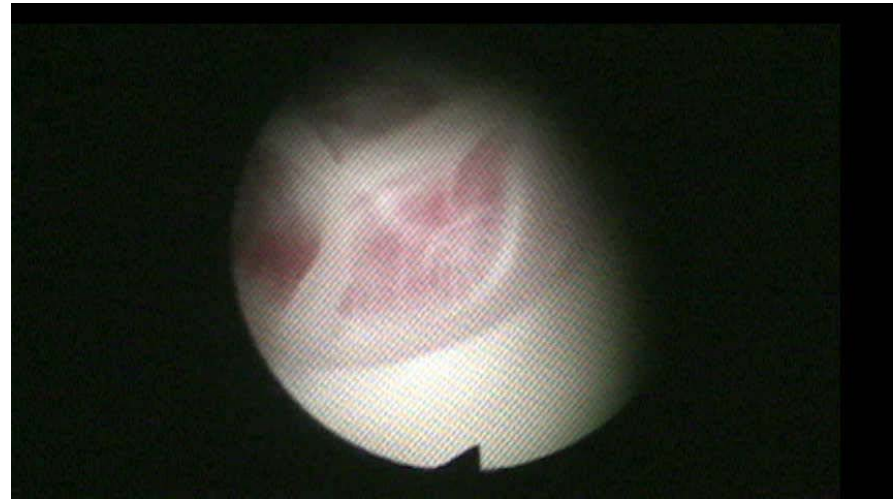

Web-video (a)

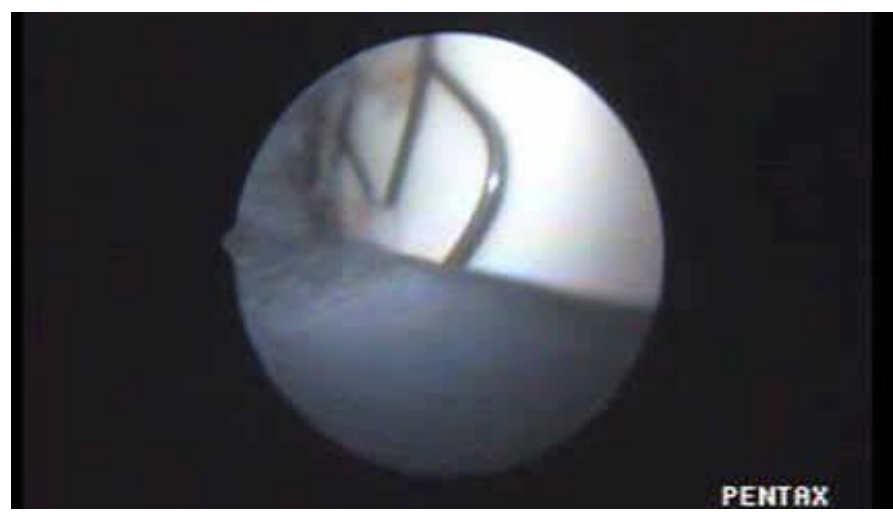

Web-video (c)

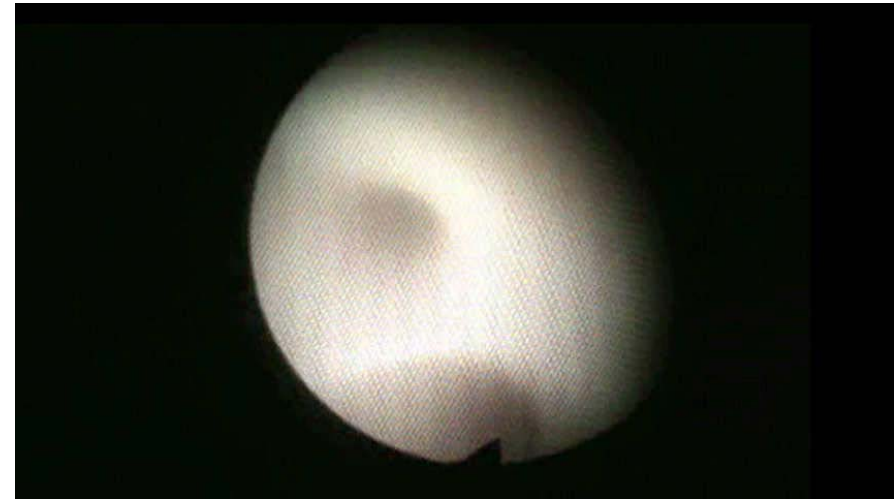

Web-video (b)

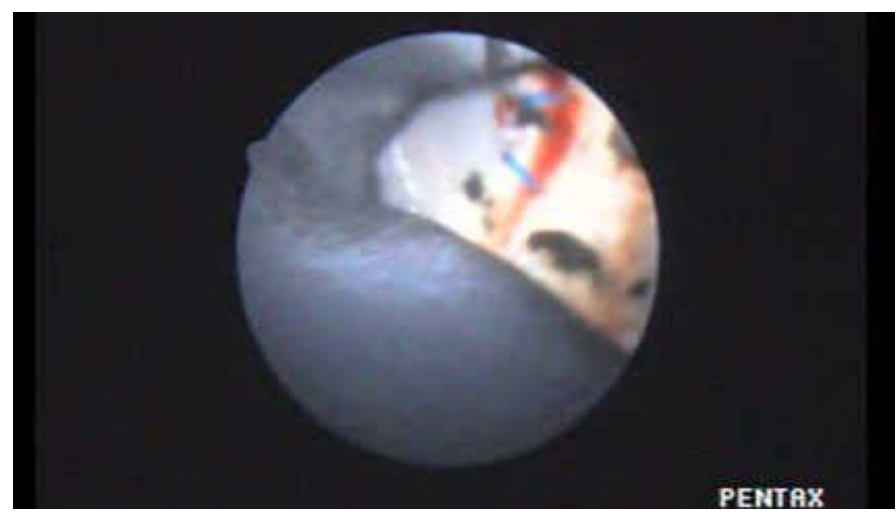

Web-video $(\mathrm{d})$

Web-videos: (a) - (d) show edited movies corresponding to (A) - (D) in Figure 3. Web-video (a) is obtained in a pig with artificially-induced ventricular bradycardia (about $1.35 \mathrm{~s}$ inter-beat interval (IBI)), while Web-videos (b) - (d) are in three different pigs with normal cardiac rhythms (approximate IBIs were $0.75 \mathrm{~s}$ in (b), 0.55 $\mathrm{s}$ in (c), and $0.8 \mathrm{~s}$ in (d)). Note that in (a) the motion of the tricuspid valve can be observed at the end of diastole and at the onset of ventricular ejection, in (b) flow streamlines forward into the brachiocephalic artery and the common carotid artery can be observed during a part of systole, in (c) the indwelling stent firmly set can be clearly observed, and in (d) there was an initial thrombosis in the suture site between the vessel and the synthetic graft, probably caused by too little injection of antithrombotic agent in this case.

\section{Conclusions}

This new method appears to be suitable for vascular diagnosis and for aiding therapeutic strategies as well as for evaluation of the curative effects in such diseases as aortic valve insufficiency, aortic dissection and aneurysm of large arteries. Technical improvements of this prototype have now been carried out for clinical use.

\section{Acknowledgments}

The authors wish to thank Professor Peter Rolfe, Harbin Institute of Technology, China, and OBH Ltd., United Kingdom, for his valuable comments on this work and his help in preparing the manuscript. A part of this study was presented at the $33^{\text {rd }}$ Annual International Conference of the IEEE Engineering in Medicine and Biology Society, Boston, USA, 2011.

\section{Role of the funding source}

This work was supported by a grant from the Regional Innovation Strategy Support Program, the Ministry of Education, Culture, Sports, Science and Technology in Japan (Period of program: from April 2008 to March 2013). The funder had no role in study design, data collection and analysis, interpretation, decision to publish, or preparation of the manuscript.

\section{Ethical conduct of studies}

The animal experiments presented herein were made under approval of Ethical Committee of Animal Experiments in Medical Department of Kanazawa University.

\section{Conflicts of interest}

The authors have declared that no competing interests exist.

\section{Authors' contributions}

- Conceived the methodology: KY.

- Designed the experiments: TY KY ST HO GY.

- Performed the experiments: ST HO KY.

- Analyzed the data: TY ST.

- Interpreted the data: TY ST HO GY KY.

- Contributed materials/analysis tools: TY ST HO GY KY.

- Drew the figure: TY ST.

- Edit the movie: TY ST.

- Wrote the paper: TY. 
Yamakoshi T (2018) A novel in vivo technique for endoscopic visualization of aortic valve motion and inner wall of large arteries for immediate postoperative direct observation

\section{References}

1. Schurr MO, Buess G, Neisius B, Voges U (2000) Robotics and telemanipulation technologies for endoscopic surgery. Surg Endosc 14: 375-381. [Crossref]

2. Kuo CS, Koch CA (1994) In vivo angioscopic visualization of right heart structure in dogs by means of a balloon-tipped fiberoptic endoscope: Potential role in percutaneous ablative procedures. Am Heart J 127: 187-197. [Crossref]

3. Horiuchi T, Masamune K, Iwase Y, Yamashita H, Tsukihara H, et al. (2011) Endoscope system with plasma flushing and coaxial round jet nozzle for off-pump cardiac surgery. Surg Endosc 25: 2296-2301. [Crossref]
4. Boese A, Sivankutty AK, Illanes A, Friebe M (2017) Intravascular endoscopy improvement through narrow-band imaging. Intern J Comput Assist Radiol and Surg 12: 2015-2021. [Crossref]

5. Gono K, Obi T, Yamaguchi M, Ohyama N, Machida H, et al. (2004) Appearance of enhanced tissue features in narrow-band endoscopic imaging. J Biomed Opt 9: 568577. [Crossref]

6. Hill BB, Hyde GL, Kuo C K, Loh FK, Wright LH, et al. (1996) Aortoscopy: A guidance system for endoluminal aortic surgery. J Vasc Surg 24: 439-448. [Crossref]

Copyright: (C2018 Yamakoshi T. This is an open-access article distributed under the terms of the Creative Commons Attribution License, which permits unrestricted use, distribution, and reproduction in any medium, provided the original author and source are credited. 